\title{
Recurrent unilateral lung ventilation disorder in a patient that experienced rocuronium-induced anaphylactic bronchospasm during laparoscopic rectal surgery
}

\section{-A case report-}

\author{
Won Joon Choi, Soo Il Choi, Jeong Min Mok, Hyun Soo Kim, and Yun Hong Kim \\ Department of Anesthesiology and Pain Medicine, Kangbuk Samsung Hospital, Sungkyunkwan University School of Medicine, \\ Seoul, Korea
}

Rocuronium is the anesthetic agent most likely to cause anaphylaxis. Immediately after intravenous rocuronium administration, the authors experienced ventilatory impairment due to unilateral bronchospasm (left lung), which was relieved by emergency treatment. However, 80 minutes after beginning laparoscopic surgery for rectal cancer, the left lung suddenly re-collapsed under pneumoperitoneum in the Trendelenburg position. A postoperative intradermal test revealed that rocuronium, vecuronium, atracurium, succinylcholine, or thiopental could induce anaphylaxis in this patient, but it was not established whether the second incident during surgery was due to endobronchial intubation or anaphylactic bronchospasm. This case cautions that under pneumoperitoneum in the Trendelenburg position, patients suspected of being prone to anaphylactic bronchospasm should also be considered at risk of endobronchial intubation. (Korean J Anesthesiol 2010; 59: 275-278)

Key Words: Anaphylaxis, Bronchial spasm, Laparoscopic surgery, Rocuronium.

Anaphylaxis is a life-threatening immune reaction mediated by IgE. It occurs during anesthesia with a incidence of 1 in 3,500 to 1 in 13,000 [1]. Rocuronium, is an aminosteroidal neuromuscular blocking agent (NMBA), has a structure similar to vecuronium and pancuronium, and has recently been reported to be the most common causative agent of anaphylaxis [1].

We experienced two complete failures of ventilation in the left lung two during the same operation; one immediately after the intravenous administration of rocuronium during anesthetic induction and the other during surgery under pneumoperitoneum in the Trendelenburg position. Here, we report and review the character of anaphylactic attributed to rocuronium, and the recurrent collapse of one lung ventilation.

Received: January 15, 2010. Revised: 1st, January 27, 2010; 2nd, February 11, 2010. Accepted: February 23, 2010.

Corresponding author: Yun Hong Kim, M.D., Department of Anesthesiology and Pain Medicine, Kangbuk Samsung Hospital, Sungkyunkwan University School of Medicine, 108, Pyeong-dong, Jongno-gu, Seoul 110-746, Korea. Tel: 82-2-2001-2322, Fax: 82-2-2001-2326, E-mail: yhkim12@yahoo.co.kr

(c) This is an open-access article distributed under the terms of the Creative Commons Attribution Non-Commercial License (http:// creativecommons.org/licenses/by-nc/3.0/), which permits unrestricted non-commercial use, distribution, and reproduction in any medium, provided the original work is properly cited. 


\section{Case Report}

A 52-year-old male, body weight $62 \mathrm{~kg}$, height $161 \mathrm{~cm}$, was planned for hand-assisted laparoscopic low anterior resection (HAL-LAR) for rectal cancer. There was no history of allergy, including asthma, atopy, or drug allergy, either for the patient himself or his family. Preoperative antibiotic sensitivity testing (AST) was negative for Cefminox. The patient received glycopyrrolate $0.2 \mathrm{mg}$ and midazolam $2 \mathrm{mg} \mathrm{IM}$ as premedication $40 \mathrm{~min}$ before surgery. Preanesthetic blood pressures (BP) and heart rates (HR) were 120/80 $\mathrm{mmHg}$ and 65 beats/min (bpm), respectively. In the supine position, endotracheal intubation was performed 1 minute after the IV administration of $1 \%$ propofol (Pofol inj, Dongkook Co., Republic of Korea) $12 \mathrm{ml}$ and rocuronium (Esmeron ${ }^{\circledR}$, NV Organon, Netherlands) $50 \mathrm{mg}$. The $7.5 \mathrm{~mm}$ ID endotracheal tube was secured at $23 \mathrm{~cm}$ at upper teeth, and equal breathing sounds were noted in both lung fields through a stethoscope. Sevoflurane- $\mathrm{N}_{2} \mathrm{O} 2 \mathrm{~L} / \mathrm{min}-\mathrm{O}_{2} 2 \mathrm{~L} / \mathrm{min}$ was then delivered for maintenance under volume controlled ventilation at a tidal volume (TV) of $600 \mathrm{ml}$ and respiratory rate (RR) of $10 \mathrm{rpm}$, at which the peak inspiratory pressure (PIP) was $20 \mathrm{mmHg}$ and end tidal carbon dioxide concentrations $\left(\mathrm{EtCO}_{2}\right)$ were $37-40 \mathrm{mmHg}$. However, 10 minutes after the administration of induction agents, BP suddenly dropped to $83 / 53 \mathrm{mmHg}$ at a HR of $145 \mathrm{bpm}$. Although BP increased to 93/58 mmHg 15 minutes after induction, capnography showed an obstructive pattern with a high PIP (41 mmHg) along with skin eruptions within the upper thorax and on both upper extremities at the same time (the severities of these were not followed after covering the body with surgical wraps during the operation). $\mathrm{EtCO}_{2}$ was maintained at $35 \mathrm{mmHg}$ at that time. On auscultation, no breathing sound was heard in the left lung field and mild wheezing was noted in the right lung. Suctioning the airway produced no secretion. We checked for proper tube placement by manual cuff palpation at the suprasternal notch, and ballottement of the cuff was strongly detected. Accordingly, we guessed anaphylactic bronchospasm due to uncertain anesthetic agents. Salbutamol sulfate was administered by inhalation three times through the endotracheal tube, but BP further declined to $75 / 40 \mathrm{mmHg}$ at a HR of $110 \mathrm{bpm}$. Skin eruptions continued to be observed within the upper thorax and both upper extremities. After discontinuing $\mathrm{N}_{2} \mathrm{O}$ and sevoflurane, piprinhydrinate $3 \mathrm{mg}$ and dexamethasone $5 \mathrm{mg}$ were injected intravenously along with salbutamol sulfate administration, but PIP and breathing sounds did not improve. Even at TV $300 \mathrm{ml}$ and RR $16 \mathrm{rpm}$, PIP remained at $30 \mathrm{mmHg}$. Although the lowest observed $\mathrm{SpO}_{2}$ value was 95\%, $\mathrm{EtCO}_{2}$ was maintained at $35 \mathrm{mmHg}$. Epinephrine $10 \mu \mathrm{g}$ and hydrocortisone $50 \mathrm{mg}$ were then administered intravenously twice and three times, respectively. Twenty minutes after the onset of symptoms, lung sounds recovered and the capnogram normalized with an $\mathrm{EtCO}_{2}$ of $36 \mathrm{mmHg}$ and a PIP of $25 \mathrm{mmHg}$ under TV $500 \mathrm{ml}$ and RR $13 \mathrm{rpm}$, and a BP and HR of 121/66 mmHg and $97 \mathrm{bpm}$, respectively.

Surgery was performed under carbon dioxide pneumoperitoneum at a pressure of $15 \mathrm{mmHg}$ in a 30 degree Trendelenburg position under pressure controlled ventilation (PCV) that limited the PIP to $27 \mathrm{mmHg}$, which demonstrated a TV of 450-500 ml. However, 80 minutes after beginning surgery under an inspiratory sevoflurane concentration of 3.6 vol\%, $\mathrm{SpO}_{2}$ suddenly decreased from $97 \%$ to $91 \%$ immediate after an intravenous injection of furosemide (Lasix ${ }^{\circledR}$, Handok, Republic of Korea) $5 \mathrm{mg}$. At that time, no auscultation sounds were detected in the left lung field under a PIP of $27 \mathrm{mmHg}$ during pressure controlled ventilation (PCV) generating a TV of only $151 \mathrm{ml}$. $\mathrm{EtCO}_{2}$ was recorded at this time at $40 \mathrm{mmHg}$. BP decreased to $80 / 50 \mathrm{mmHg}$ with a HR of $75 \mathrm{bpm}$. Thus, salbutamol sulfate inhalation and intravenous hydrocortisone and epinephrine were re-challenged, and the posture changed from Trendelenburg to supine. 10 minutes later, $\mathrm{SpO}_{2}$ and $\mathrm{BP}$ recovered to $96 \%$ and $153 / 94 \mathrm{mmHg}$, respectively, and both lung sounds were fully regained. The surgery was completed after colostomy. Total anesthetic duration was 200 minutes. The postoperative chest radiograph was normal, and the endotracheal tube was extubated 9 hours after completing surgery.

Patch, skin prick, and intradermal tests (IDT) were carried out on postoperative days 8,15 , and 16 to confirm the relationships between the patient's symptoms and anesthetic agents. Several types of anesthetics were examined (Table 1). All skin tests were performed on the patient's back. The concentrations of test drugs used depended on the type of test. As for the patch test, pure commercialized drugs were applied. Skin prick tests were

Table 1. Results of Anesthetic Skin Reactivity Tests

\begin{tabular}{lccc}
\hline \multicolumn{1}{c}{ Drug } & Patch test & Skin prick test & Intradermal test \\
\hline Rocuronium & - & & + \\
Atracurium & - & - & ++ \\
Vecuronium & - & - & ++ \\
Succinylcholine & - & - & ++ \\
Propofol & - & - & - \\
Thiopental sodium & - & - & + \\
Glycopyrrolate & - & - & - \\
Pyridostigmine & - & & - \\
Hyperbaric bupivacaine & - & & - \\
Lidocaine & - & & - \\
Bupivacaine & - & & - \\
Latex & - & & - \\
\hline
\end{tabular}

The results were observed 15 minutes after applying the drugs. + : a wheal of 3-5 mm with surrounding erythema of 5-10 $\mathrm{mm},++:$ a wheal $>5 \mathrm{~mm}$ with erythema of $>10 \mathrm{~mm}$. Blank means that the test was not performed. 
performed using solutions of the test drugs, diluted sequentially $\left(10^{-2}\right.$ and $\left.10^{-1}\right)$ in $0.9 \%$ saline and IDTs were conducted using a $10^{-1}$ dilution. Skin test results were graded + when a wheal of 3-5 mm with surrounding erythema of 5-10 $\mathrm{mm}$ arose within 15 minutes after injection, and as ++ when a wheal of $>5 \mathrm{~mm}$ with erythema of $>10 \mathrm{~mm}$ appeared within 15 minutes. It was found that rocuronium, succinylcholine, atracurium, vecuronium, and thiopental sodium produced a positive reaction only by IDT (Table 1).

Surgery to repair the colostomy was carried out 50 days later under spinal anesthesia using $0.5 \%$ hyperbaric bupivacaine $15 \mathrm{mg}$, which had shown no reactivity on previous skin tests. The anesthetic duration was 90 minutes and no problem was experienced perioperatively.

\section{Discussion}

The anaphylactic symptoms caused by rocuronium are variable, and include; tachycardia, severe hypotension, erythematous skin rash, facial or generalized edema, and bronchospasm [2,3]. Although anaphylactic symptoms usually occur immediately after an allergen challenge, they can be delayed for 2-20 minutes [4,5] About $70 \%$ of patients that experience an anaphylactic bronchospasm have accompanying cutaneous signs, such as, a rash or flush [6]. The symptom course during induction in our case was very similar to that reported for rocuronium-induced anaphylaxis.

The clinical aspects of unilateral bronchospasm are similar to those of endobronchial intubation [7]. However, endobronchial intubation is also a risk factor of reflex bronchospasm because airway instrumentation near the carina, which contains abundant irritant receptors, can cause reflex bronchoconstriction [6]. However, reflex bronchospasm after intubation is not accompanied by cutaneous reactions because it does not occur via an immune pathway [8]. Propofol can cause skin rashes irrespective of anaphylaxis, but for non-immune reactions, skin reactions are not associated with cardiovascular deteriorations [9]. Accordingly, in our case, it is unlikely that the first ventilation disorder was caused by airway irritation, or that the skin eruptions were related to propofol induced nonimmune reactions.

It has been reported that the mean distance between the carina and the endotracheal tube tip in the supine position is $3.37 \pm 2.21 \mathrm{~cm}$ when palpating cuff ballottement at the suprasternal notch [10]. Thus, the possibility of endobronchial intubation could be excluded by manual cuff palpation at the sternal notch in the supine position during the first ventilation disorder. Furthermore, a unilateral silent lung accompanied by a sudden decrease in BP suggested immune-related bronchospasm rather than endobronchial intubation. Thus, given this information and the skin test results, we were able to strongly associate the first incident with rocuronium-induced anaphylactic bronchospasm. Intradermal testing is valuable in terms of discerning whether a suspected perioperative allergic reaction is caused by IgE-mediated immediate hypersensitivity, especially that due to anesthetics [11].

Unilateral bronchospasm usually occurs due to topical irritation of the respiratory system $[12,13]$. However, unilateral bronchospasm in our case was triggered by the systemic effects of intravenous agents. Tsubo et al. [5] suggested that latex allergen, which is absorbed into systemic circulation during intra-abdominal gynecological surgery, might induce unilateral bronchospasm. Thus, it is possible that anaphylactic antigens can induce unilateral bronchospasm not only by topical irritation of the involved lung, but also rarely via systemic effects.

However, contrary to the first bronchospasm, chances were that other risk factors might influence on happening of the second case of unilateral silent breath sound. The recurrence of anaphylactic symptoms is not common after successful resuscitation [2,3], and it our case, the Trendelenburg position itself is considered unlikely trigger factor because NMBAsinduced anaphylactic hypotension used to be resuscitated by placing patients in the Trendelenburg position. However, it is possible that pneumoperitoneum in the Trendelenburg position might have induced unilateral endobronchial intubation [14]. Furthermore, a zero risk of endobronchial intubation in the Trendelenburg position under pneumoperitoneum is not guaranteed by confirming tube placement by manual cuff palpation in the supine position [14]. However, we did not recheck the cuff ballottement at the sternal notch after changing posture, and thus, it is not clear whether the second incident was caused by bronchospasm or a malpositioned tube.

Moreover, although furosemide is known to be a causative agent of anaphylaxis [15], unilateral ventilatory failure was unlikely to been related to the administration of furosemide, because skin tests were negative.

In conclusion, the intravenous administration of rocuronium can induce unilateral bronchospasm. Nevertheless, although a patient may suffer unilateral bronchospasm due to anaphylaxis, it should also be considered that recurrent unilateral ventilatory failure might be induced during the same surgery by endobronchial intubation when a patient under pneumoperitoneum is in the Trendelenburg position.

\section{References}

1. Hepner DL, Castells MC. Anaphylaxis during the perioperative period. Anesth Analg 2003; 97: 1381-95.

2. Baillard C, Korinek AM, Galanton V, Le Manach Y, Larmignat P, 
Cupa M, et al. Anaphylaxis to rocuronium. Br J Anaesth 2002; 88: 600-2.

3. Lee HM, Song SO. Anaphylaxis after injection of rocuronium. Korean J Anesthesiol 2006; 51: 101-4.

4. Roizen MF, Fleisher LA. Anesthetic implications of concurrent diseases. In: Miller's Anesthesia. 7th ed. Edited by Miller RD: Philadelphia, Churchil Livingstone Elsevier. 2010, p 1110.

5. Tsubo T, Suzuki A, Okawa H, Ishihara H, Oishi T, Ota S, et al. A case of intraoperative unilateral bronchospasm due to possible latex anaphylaxis. J Clin Anesth (Jpn) 1999; 23: 1631-2.

6. Westhorpe RN, Ludbrook GL, Helps SC. Crisis management during anaesthesia: bronchospasm. Qual Saf Health Care 2005; 14: e7.

7. Gal TJ. Physiologic and therapeutic concerns in anesthesia for patients with reactive airways. Semin Anesth Perioperat Med Pain 1996; 15: 363-75.

8. Zeidan A, Nahle N, Baraka A. Does bilateral thoracic sympathectomy predispose to reflex bronchospasm following tracheal intubation? Can J Anaesth 2005; 52: 997-8.
9. Redfern N, Stafford MA, Hull CJ. Incremental propofol for short procedures. Br J Anaesth 1985; 57: 1178-82.

10. Ledrick D, Plewa M, Casey K, Taylor J, Buderer N. Evaluation of manual cuff palpation to confirm proper endotracheal tube depth. Prehosp Disaster Med 2008; 23: 270-4.

11. Kranke B, Aberer W. Skin testing for IgE-mediated drug allergy. Immunol Allergy Clin North Am 2009; 29: 503-16.

12. Narr BJ, Fromme GA, Peters SG. Unilateral bronchospasm during pleurodesis in an asthmatic patient. Chest 1990; 98: 767-8.

13. Farmery AD. Severe unilateral bronchospasm mimicking inadvertent endobronchial intubation: a complication of the use of a topical lidocaine Laryngojet injector. Br J Anaesth 2000; 85: 917-9.

14. Lobato EB, Paige GB, Brown MM, Bennett B, Davis JD. Pneumoperitoneum as a risk factor for endobronchial intubation during laparoscopic gynecologic surgery. Anesth Analg 1998; 86: 301-3.

15. Hansbrough JR, Wedner HJ, Chaplin DD. Anaphylaxis to intravenous furosemide. J Allergy Clin Immunol 1987; 80: 538-41. 